University of Warwick institutional repository: http://go.warwick.ac.uk/wrap This paper is made available online in accordance with publisher policies. Please scroll down to view the document itself. Please refer to the repository record for this item and our policy information available from the repository home page for further information.

To see the final version of this paper please visit the publisher's website. Access to the published version may require a subscription.

Author(s): PETER WALTERS

Article Title: REGULARITY CONDITIONS AND BERNOULLI

PROPERTIES OF EQUILIBRIUM STATES AND \$g\$-MEASURES

Year of publication: 2005

Link to published

version: http://dx.doi.org/10.1112/S0024610704006076

Publisher statement: None 


\title{
REGULARITY CONDITIONS AND BERNOULLI PROPERTIES OF EQUILIBRIUM STATES AND $g$-MEASURES
}

\author{
PETER WALTERS
}

\begin{abstract}
When $T: X \longrightarrow X$ is a one-sided topologically mixing subshift of finite type and $\varphi: X \longrightarrow R$ is a continuous function, one can define the Ruelle operator $\mathcal{L}_{\varphi}: C(X) \longrightarrow C(X)$ on the space $C(X)$ of real-valued continuous functions on $X$. The dual operator $\mathcal{L}_{\varphi}^{*}$ always has a probability measure $\nu$ as an eigenvector corresponding to a positive eigenvalue $\left(\mathcal{L}_{\varphi}^{*} \nu=\lambda \nu\right.$ with $\left.\lambda>0\right)$. Necessary and sufficient conditions on such an eigenmeasure $\nu$ are obtained for $\varphi$ to belong to two important spaces of functions, $W(X, T)$ and $\operatorname{Bow}(X, T)$. For example, $\varphi \in \operatorname{Bow}(X, T)$ if and only if $\nu$ is a measure with a certain approximate product structure. This is used to apply results of Bradley to show that the natural extension of the unique equilibrium state $\mu_{\varphi}$ of $\varphi \in \operatorname{Bow}(X, T)$ has the weak Bernoulli property and hence is measure-theoretically isomorphic to a Bernoulli shift. It is also shown that the unique equilibrium state of a two-sided Bowen function has the weak Bernoulli property. The characterizations mentioned above are used in the case of $g$-measures to obtain results on the 'reverse' of a $g$-measure.
\end{abstract}

\section{Introduction}

We consider subshifts of finite type with a finite number of symbols. Let $k \geqslant 2$, and let $\Gamma=\{1,2, \ldots, k\}$ be the set of symbols. Let $A=\left(a_{i j}\right)$ be a $k \times k$ matrix with each entry $a_{i j} \in\{0,1\}$ and with no zero row and no zero column.

Let

$$
X_{A}=\left\{x=\left(x_{n}\right)_{n=0}^{\infty} \in \prod_{0}^{\infty} \Gamma \mid a_{x_{n} x_{n+1}}=1 \forall n \geqslant 0\right\}
$$

and

$$
\hat{X}_{A}=\left\{x=\left(x_{n}\right)_{n=-\infty}^{\infty} \in \prod_{-\infty}^{\infty} \Gamma \mid a_{x_{n} x_{n+1}}=1 \forall n \in Z\right\} .
$$

Both are compact sets under the product topologies on $\prod_{0}^{\infty} \Gamma$ and $\prod_{-\infty}^{\infty} \Gamma$ when $\Gamma$ is equipped with the discrete topology. The one-sided subshift of finite type determined by $A$ is the continuous surjection $T: X_{A} \longrightarrow X_{A}$ defined by $T\left(\left(x_{0}, x_{1}, \ldots\right)\right)=$ $\left(x_{1}, x_{2}, \ldots\right)$. The two-sided subshift of finite type determined by $A$ is the homeomorphism $S: \hat{X}_{A} \longrightarrow \hat{X}_{A}$ defined by

$$
S\left(\left(\ldots x_{-1} \stackrel{*}{x_{0}} x_{1} \ldots\right)\right)=\left(\ldots x_{-1} x_{0} \stackrel{*}{x_{1}} x_{2} \ldots\right),
$$

where the symbol $*$ is over the 0th position. Both shifts are called topologically mixing if there exists $M \geqslant 1$ with the product matrix $A^{M}>0$, that is, every entry of $A^{M}$ is non-zero. We use $M$ for such a number throughout the paper. This is equivalent to $T$ being topologically mixing (that is, for all non-empty open sets $U, V$, there exists $M \geqslant 1$ with $U \cap T^{-n} V \neq \varnothing$ for all $\left.n \geqslant M\right)$, and to

Received 3 July 2003; revised 15 May 2004.

2000 Mathematics Subject Classification 27D35 (primary), 28D20, 37A30, 37B10 (secondary). 
$S$ being topologically mixing. If $p \leqslant q$ and $b_{p}, \ldots, b_{q} \in \Gamma$, then ${ }_{p}\left[b_{p}, \ldots, b_{q}\right]=$ ${ }_{p}\left[b_{p}, \ldots, b_{q}\right]_{q}=\left[b_{p}, \ldots, b_{q}\right]_{q}=\left\{x \in X_{A} \mid x_{i}=b_{i}\right.$ for $\left.p \leqslant i \leqslant q\right\}$ when $0 \leqslant p$, and also ${ }_{p}\left[b_{p}, \ldots, b_{q}\right]={ }_{p}\left[b_{p}, \ldots, b_{q}\right]_{q}=\left[b_{p}, \ldots, b_{q}\right]_{q}=\left\{x \in \hat{X}_{A} \mid x_{i}=b_{i}\right.$ for $\left.p \leqslant i \leqslant q\right\}$ when $p \in Z$. For $n, p \geqslant 1$, we have $T^{p}\left({ }_{0}\left[x_{0}, \ldots, x_{p+n-1}\right]\right)={ }_{0}\left[x_{p}, \ldots, x_{p+n-1}\right]$ if ${ }_{0}\left[x_{0}, \ldots, x_{p+n-1}\right] \neq \varnothing$, and $T^{-p}\left({ }_{0}\left[x_{0}, \ldots, x_{n-1}\right]\right)={ }_{p}\left[x_{0}, \ldots, x_{n-1}\right]$. An allowable block in $X_{A}$ or $\hat{X}_{A}$ is a string $b_{0}, \ldots, b_{n-1}$ of symbols with $a_{b_{0} b_{1}} a_{b_{1} b_{2}} \ldots a_{b_{n-2} b_{n-1}}=1$. If $b_{0}, \ldots, b_{n-1}$ is an allowable block, then we call ${ }_{p}\left[b_{0}, \ldots, b_{n-1}\right]$ an allowable cylinder. If $b, c \in \Gamma$ and $p, q \in N$, then $b^{p} c^{q}$ denotes the block $b_{0}, \ldots, b_{p+q-1}$, where $b_{i}=b$ for $0 \leqslant i \leqslant p-1$ and $b_{i}=c$ for $p \leqslant i \leqslant p+q-1$. If $x_{0}, \ldots, x_{n-1}$ is an allowable block in $X_{A}$ and $z=\left(z_{0}, z_{1}, \ldots\right) \in X_{A}$ with $a_{x_{n-1} z_{0}}=1$, then $\left(x_{0}, \ldots, x_{n-1}, z\right)$ denotes the member $y=\left(y_{i}\right)$ of $X_{A}$ with $y_{i}=x_{i}$ for $0 \leqslant i \leqslant n-1$ and $y_{i+n}=z_{i}$ for $i \geqslant 0$.

We often write $X$ and $\hat{X}$ for $X_{A}$ and $\hat{X}_{A}$. Consider a subshift of finite type (SFT) $T: X \longrightarrow X$. We use $C(X)$ to denote the space of all real-valued continuous functions on $X$, equipped with the supremum norm. We let $M(X)$ denote the space of all probability measures on the Borel subsets of $X$, equipped with the weak*-topology, and let $M(X, T)$ denote the non-empty subset of $T$ invariant members of $M(X)$. We say that $\tau \in M(X)$ has support $X$ if $\tau(U)>0$ for every non-empty open set $U$. If $\varphi \in C(X)$, we let $P(T, \varphi)$ denote the pressure of $T$ at $\varphi[\mathbf{1 3}]$, and let $T_{n} \varphi$ be the function $\sum_{i=0}^{n-1} \varphi \circ T^{i}$. Similar notation applies to $S: \hat{X} \longrightarrow \hat{X}$. When $T$ is a one-sided subshift of finite type, the Ruelle operator of $\varphi \in C(X)$ is denoted by $\mathcal{L}_{\varphi}: C(X) \longrightarrow C(X)$, so that $\left(\mathcal{L}_{\varphi} f\right)(x)=\sum e^{\varphi(y)} f(y)$, where the sum is over all $y \in T^{-1} x$. The dual operator $\mathcal{L}_{\varphi}^{*}$ always has an eigenmeasure in $M(X)$, that is, there exists $\nu \in M(X)$ and $\lambda>0$ with $\mathcal{L}_{\varphi}^{*} \nu=\lambda \nu$. For $\varphi \in C(X)$ and $T$ a one-sided subshift of finite type, we define $v_{n}(\varphi)$, for $n \geqslant 1$, as $v_{n}(\varphi)=\sup \left\{\varphi(x)-\varphi\left(x^{\prime}\right) \mid x, x^{\prime} \in X\right.$ and $\left.x_{i}=x_{i}^{\prime}, 0 \leqslant i \leqslant n-1\right\}$. We define the space $\operatorname{Bow}(X, T)$ to be $\left\{\varphi \in C(X) \mid \sup _{n \geqslant 1} v_{n}\left(T_{n} \varphi\right)<\infty\right\}$ and the space $W(X, T)$ to be $\left\{\varphi \in C(X) \mid \sup _{n \geqslant 1} v_{n+p}\left(T_{n} \varphi\right) \rightarrow 0\right.$ as $\left.p \rightarrow \infty\right\}[\mathbf{1 4}, \mathbf{1 5}]$. We have $W(X, T) \subset \operatorname{Bow}(X, T)$, because $\varphi \in \operatorname{Bow}(X, T)$ if and only if there is some $p \geqslant 0$ with $\sup _{n \geqslant 1} v_{n+p}\left(T_{n} \varphi\right)<\infty$. If $\varphi \in C(X)$ has summable variations (that is, $\left.\sum_{n=1}^{\infty} v_{n}(\varphi)<\infty\right)[\mathbf{1 1}]$, then $\varphi \in W(X, T)$.

In $[\mathbf{1 2}]$, the author showed that for a topologically mixing subshift of finite type (TMSFT), if $\varphi \in W(X, T)$, then the Ruelle operator theorem holds (that is, there exists $\lambda>0, \nu \in M(X)$, and $h \in C(X)$ with $h>0$ and $\int h d \nu=1$ such that $\mathcal{L}_{\varphi} h=\lambda h$, $\mathcal{L}_{\varphi}^{*} \nu=\lambda \nu$ and for all $f \in C(X)$,

$$
\frac{\left(\mathcal{L}_{\varphi}^{n} f\right)(x)}{\lambda^{n}} \rightrightarrows h(x) \int f d \nu,
$$

where $\rightrightarrows$ denotes uniform convergence on $X), \varphi$ has a unique equilibrium state $\mu_{\varphi}$ and $\left(T, \mu_{\varphi}\right)$ has a Bernoulli natural extension. Here $\mu_{\varphi}=h \nu$, and $\mu_{\varphi}$ is the unique $g$-measure for the $g$-function $g(x)=e^{\varphi(x)} h(x) / \lambda h(T x)$. In [14], the author considered these questions for $\varphi \in \operatorname{Bow}(X, T)$ and proved a weakened version of the Ruelle operator theorem. Each $\varphi \in \operatorname{Bow}(X, T)$ has a unique equilibrium state $\mu_{\varphi}$. We show in this paper that $\left(T, \mu_{\varphi}\right)$ has a Bernoulli natural extension. We obtain necessary and sufficient conditions on an eigenmeasure $\nu$ for $\varphi \in C(X)$ to ensure that $\varphi \in \operatorname{Bow}(X, T)$ and to ensure that $\varphi \in W(X, T)$. These give characterizations of when $\varphi \in \operatorname{Bow}(X, T)$ in terms of $\mu_{\varphi}$. When this is applied to the cases $\varphi=\log g$ and $g$ is a $g$-function (see Section 3), we obtain results about the 'reverse' of a $g$-measure. 
For a two-sided topologically mixing subshift of finite type, $S: \hat{X} \longrightarrow \hat{X}$ and $\hat{\varphi} \in C(\hat{X})$, we let $\operatorname{var}_{n}(\hat{\varphi})$, for $n \geqslant 1$, denote $\operatorname{var}_{n}(\hat{\varphi})=\sup \left\{\hat{\varphi}(x)-\hat{\varphi}\left(x^{\prime}\right) \mid x, x^{\prime} \in \hat{X}\right.$ and $x_{i}=x_{i}^{\prime}$ for $\left.\left.-(n-1) \leqslant i \leqslant n-1\right)\right\}$. Then let $\operatorname{Bow}\left(\hat{X}_{A}, S\right)=\{\hat{\varphi} \in C(\hat{X}) \mid$ $\left.\sup _{n \geqslant 1} \operatorname{var}_{n}\left(S_{n} \hat{\varphi}\right)<\infty\right\}$, where $S_{n} \hat{\varphi}=\sum_{i=0}^{n-1} \hat{\varphi} \circ S^{i}$. Bowen showed that each $\hat{\varphi} \in$ $\operatorname{Bow}\left(\hat{X}_{A}, S\right)$ has a unique equilibrium state $\hat{\mu}_{\hat{\varphi}}[\mathbf{2}]$. We show that $\left(S, \hat{\mu}_{\varphi}\right)$ is isomorphic to a Bernoulli shift.

\section{Eigenmeasures of the Ruelle operator}

Lemma 1.1. Let $T: X \longrightarrow X$ be a one-sided topologically mixing subshift of finite type, let $\varphi \in C(X)$, and let $\nu \in M(X)$ and $\lambda>0$ satisfy $\mathcal{L}_{\varphi}^{*} \nu=\lambda \nu$. Then for all $n \geqslant 1, p \geqslant 1$ and $x \in X$, we have

$$
\nu\left({ }_{0}\left[x_{0}, \ldots, x_{n+p-1}\right]\right)=\frac{1}{\lambda^{p}} \int_{z \in_{0}\left[x_{p}, \ldots, x_{n+p-1}\right]} e^{\left(T_{p} \varphi\right)\left(x_{0}, \ldots, x_{p-1}, z\right)} d \nu(z) .
$$

Proof.

$$
\begin{aligned}
\nu\left({ }_{0}\left[x_{0}, \ldots, x_{n+p-1}\right]\right) & =\frac{1}{\lambda^{p}} \int_{X} \mathcal{L}_{\varphi}^{p} \chi_{0}\left[x_{0}, \ldots, x_{n+p-1}\right](z) d \nu(z) \\
& =\frac{1}{\lambda^{p}} \int_{0\left[x_{p}, \ldots, x_{n+p-1}\right]} e^{\left(T_{p} \varphi\right)\left(x_{0}, \ldots, x_{p-1}, z\right)} d \nu(z) .
\end{aligned}
$$

In part (i) of the next result, the number $k$ is the number of symbols in the topologically mixing subshift of finite type and $M$ is a natural number for which the $M$ th power $A^{M}$ of the transition matrix $A$ has every entry non-zero.

Theorem 1.2. Let $T: X \longrightarrow X$ be a one-sided topologically mixing subshift of finite type and let $\varphi \in C(X)$. Let $\nu \in M(X)$ and $\lambda>0$ satisfy $\mathcal{L}_{\varphi}^{*} \nu=\lambda \nu$. Then $\lambda=e^{P(T, \varphi)}, \nu$ is a supported measure, and we have the following.

(i) For all $p \geqslant 1$ and for all $x \in X$,

$$
\left(e^{v_{p}\left(T_{p} \varphi\right)} \lambda^{M} e^{M\|\varphi\|}\right)^{-1} \leqslant \frac{\nu\left({ }_{0}\left[x_{0}, \ldots, x_{p-1}\right]\right) \lambda^{p}}{e^{\left(T_{p} \varphi\right)(x)}} \leqslant k^{M} e^{v_{p}\left(T_{p} \varphi\right)} \frac{e^{M\|\varphi\|}}{\lambda^{M}} .
$$

(ii) For all $n \geqslant 1, p \geqslant 1$ and for all $x \in X$,

$$
e^{-v_{n+p}\left(T_{p} \varphi\right)} \leqslant \frac{\nu\left({ }_{0}\left[x_{0}, \ldots, x_{n+p-1}\right]\right)}{\nu\left({ }_{0}\left[x_{p}, \ldots, x_{n+p-1}\right]\right)} \frac{\lambda^{p}}{e^{\left(T_{p} \varphi\right)(x)}} \leqslant e^{v_{n+p}\left(T_{p} \varphi\right)} .
$$

(iii) The measures $\nu$ and $\nu \circ T^{-p}$ are equivalent and

$$
\frac{d \nu \circ T^{-p}}{d \nu}=\frac{\mathcal{L}_{\varphi}^{p} 1}{\lambda^{p}}
$$

(iv) For each symbol $i \in\{1,2, \ldots, k\},\left.T\right|_{0}[i]$ is injective, so $\left.\nu \circ T\right|_{0}[i]$ is a measure on $0[i]$ and

$$
\frac{d\left(\left.\nu \circ T\right|_{0}[i]\right)}{d\left(\left.\nu\right|_{0[i]}\right)}=\lambda e^{-\varphi}
$$

on $0[i]$. 
(v) For all $n \geqslant 1, p \geqslant 1$ and for all $w \in X$,

$$
e^{-v_{n}\left(\log \mathcal{L}_{\varphi}^{p} 1\right)} \leqslant \frac{\nu\left({ }_{p}\left[w_{0}, \ldots, w_{n-1}\right]\right)}{\nu\left({ }_{0}\left[w_{0}, \ldots, w_{n-1}\right]\right)} \frac{\lambda^{p}}{\left(\mathcal{L}_{\varphi}^{p} 1\right)(w)} \leqslant e^{v_{n}\left(\log \mathcal{L}_{\varphi}^{p} 1\right)} .
$$

Proof. (i)

$$
\begin{aligned}
\nu\left({ }_{0}\left[x_{0}, \ldots, x_{p-1}\right]\right) & =\int_{X} \chi_{0}\left[x_{0}, \ldots, x_{p-1}\right](z) d \nu(z) \\
& =\frac{1}{\lambda^{p+M}} \int_{X} \mathcal{L}_{\varphi}^{p+M} \chi_{0}\left[x_{0}, \ldots, x_{p-1}\right](z) d \nu(z) .
\end{aligned}
$$

Now

$$
\mathcal{L}_{\varphi}^{p+M} \chi_{0\left[x_{0}, \ldots, x_{p-1}\right]}(z)=\sum e^{\left(T_{p+M} \varphi\right)\left(x_{0}, \ldots, x_{p-1}, b_{0}, \ldots, b_{M-1}, z\right)},
$$

where the sum is over all $b_{0}, \ldots, b_{M-1}$ with the product $a_{x_{p-1} b_{0}} a_{b_{0} b_{1}} \ldots a_{b_{M-1} z_{0}}>0$. For any $x_{p-1}, z_{0}$, such $\left(b_{0}, \ldots, b_{M-1}\right)$ exist by topological mixing, and there are at most $k^{M}$ choices. For each such admissable $\left(b_{0}, \ldots, b_{M-1}\right)$, we have $\left|\left(T_{p+M} \varphi\right)\left(x_{0}, \ldots, x_{p-1}, b_{0}, \ldots, b_{M-1}, z\right)-\left(T_{p} \varphi\right)(x)\right| \leqslant v_{p}\left(T_{p} \varphi\right)+M\|\varphi\|$, so we get the inequalities in (i).

The left-hand inequality in (i) shows that $\nu$ is supported. To see that $\lambda=e^{P(T, \varphi)}$, we use the fact that $(1 / p) \log \left(\mathcal{L}_{\varphi}^{p} 1\right)(x) \rightrightarrows P(T, \varphi)[\mathbf{1 4}$, Theorem 1.3]. If $\varepsilon>0$, then there exists $N_{\varepsilon}$ with $e^{p P(T, \varphi)-p \varepsilon} \leqslant\left(\mathcal{L}_{\varphi}^{p} 1\right)(x) \leqslant e^{p P(T, \varphi)+p \varepsilon}$ for all $x \in X$ and for all $p \geqslant N_{\varepsilon}$. Integrating with respect to $\nu$ gives $e^{p(P(T, \varphi)-\varepsilon)} \leqslant \lambda^{p} \leqslant e^{p(P(T, \varphi)+\varepsilon)}$ for all $p \geqslant N_{\varepsilon}$. Hence $e^{P(T, \varphi)-\varepsilon} \leqslant \lambda \leqslant e^{P(T, \varphi)+\varepsilon}$, and since this holds for every $\varepsilon>0$, we have $\lambda=e^{P(T, \varphi)}$.

(ii) Statement (ii) follows from Lemma 1.1 and the inequality $\mid\left(T_{p} \varphi\right)\left(x_{0}, \ldots\right.$, $\left.x_{p-1} z\right)-\left(T_{p} \varphi\right)(x) \mid \leqslant v_{n+p}\left(T_{p} \varphi\right)$, when $z \in_{0}\left[x_{p}, \ldots, x_{n+p-1}\right]$.

(iii) Fix a cylinder ${ }_{0}\left[w_{0}, \ldots, w_{p-1}\right]$ in $X$. Then

$$
T^{-p}\left({ }_{0}\left[w_{0}, \ldots, w_{n-1}\right]\right)=\bigcup_{0}\left[y_{0}, \ldots, y_{p-1}, w_{0}, \ldots, w_{n-1}\right]
$$

where the union is over all $\left(y_{0}, \ldots, y_{p-1}\right)$ with $a_{y_{0} y_{1}} \ldots a_{y_{p-1} w_{0}}=1$, and this is a disjoint union. Apply Lemma 1.1 to each such admissable ${ }_{0}\left[y_{0}, \ldots, y_{p-1}\right.$, $\left.w_{0}, \ldots, w_{n-1}\right]$ to get

$$
\nu\left({ }_{0}\left[y_{0}, \ldots, y_{p-1}, w_{0}, \ldots, w_{n-1}\right]\right)=\frac{1}{\lambda^{p}} \int_{z \in_{0\left[w_{0}, \ldots, w_{n-1}\right]}} e^{\left(T_{p} \varphi\right)\left(y_{0}, \ldots, y_{p-1}, z\right)} d \nu(z) .
$$

Now sum over all $\left(y_{0}, \ldots, y_{p-1}\right)$ to get

$$
\left(\nu \circ T^{-p}\right)\left({ }_{0}\left[w_{0}, \ldots, w_{n-1}\right]\right)=\int_{z \in_{0}\left[w_{0}, \ldots, w_{n-1}\right]} \frac{\left(\mathcal{L}_{\varphi}^{p} 1\right)(z)}{\lambda^{p}} d v(z) .
$$

(iv) We have to show that for each cylinder ${ }_{0}\left[i, i_{1}, \ldots, i_{p-1}\right]$, we have

$$
\nu\left(T_{0}\left[i, i_{1}, \ldots, i_{p-1}\right]\right)=\lambda \int_{x \in \in_{0}\left[i, i_{1}, \ldots, i_{p-1}\right]} e^{-\varphi(x)} d \nu(x) .
$$


We have

$$
\begin{aligned}
& \lambda \int_{{ }_{0}\left[i, i_{1}, \ldots, i_{p-1}\right]} e^{-\varphi(x)} d \nu(x)=\lambda \int_{X} e^{-\varphi} \chi_{0}\left[i, i_{1}, \ldots, i_{p-1}\right] d \nu \\
& =\int_{X} \mathcal{L}_{\varphi}\left(e^{-\varphi} \chi_{0}\left[i, i_{1}, \ldots, i_{p-1}\right]\right) d \nu \\
& =\int_{X} \chi_{0}\left[i, i_{1}, \ldots, i_{p-1}\right](i z) d \nu(z) \\
& =\nu\left(T_{0}\left[i, i_{1}, \ldots, i_{p-1}\right]\right) .
\end{aligned}
$$

(v) By (iii),

$$
\nu\left({ }_{p}\left[w_{0}, \ldots, w_{n-1}\right]\right)=\int_{z \in_{0}\left[w_{0}, \ldots, w_{n-1}\right]} \frac{\mathcal{L}_{\varphi}^{p} 1}{\lambda^{p}}(z) d \nu(z) .
$$

If $w \in X$ and $z \in{ }_{0}\left[w_{0}, \ldots, w_{n-1}\right]$, then

$$
e^{-v_{n}\left(\log \mathcal{L}_{\varphi}^{p} 1\right)} \leqslant \frac{\left(\mathcal{L}_{\varphi}^{p} 1\right)(z)}{\left(\mathcal{L}_{\varphi}^{p} 1\right)(w)} \leqslant e^{v_{n}\left(\log \mathcal{L}_{\varphi}^{p} 1\right)}
$$

so (v) holds.

Recall that we use the symbol $\rightrightarrows$ to denote uniform convergence on $X$.

Corollary 1.3. Let $T: X \longrightarrow X$ be a one-sided topologically mixing subshift of finite type, and let $\varphi \in C(X)$. Let $\nu \in M(X)$ and let $\lambda>0$ satisfy $\mathcal{L}_{\varphi}^{*} \nu=\lambda \nu$. For each fixed $p \geqslant 1$,

$$
\frac{\nu\left({ }_{0}\left[x_{0}, \ldots, x_{n+p-1}\right]\right)}{\nu\left({ }_{0}\left[x_{p}, \ldots, x_{n+p-1}\right]\right)} \rightrightarrows \frac{e^{\left(T_{p} \varphi\right)(x)}}{\lambda^{p}} \quad \text { as } n \rightarrow \infty,
$$

and

$$
\frac{\nu\left({ }_{p}\left[w_{0}, \ldots, w_{n-1}\right]\right)}{\nu\left({ }_{0}\left[w_{0}, \ldots, w_{n-1}\right]\right)} \rightrightarrows \frac{\left(\mathcal{L}_{\varphi}^{p} 1\right)(w)}{\lambda^{p}} \quad \text { as } n \rightarrow \infty .
$$

Proof. The first statement is by Theorem 1.2(ii), since, for $p$ fixed, $v_{n+p}\left(T_{p} \varphi\right) \rightarrow 0$ as $n \rightarrow \infty$, and the second statement is by Theorem $1.2(\mathrm{v})$, since, for fixed $p, v_{n}\left(\log \mathcal{L}_{\varphi}^{p} 1\right) \rightarrow 0$ as $n \rightarrow \infty$.

In fact, the case $p=1$ in the first statement of Corollary 1.3 gives

$$
\frac{\nu\left({ }_{0}\left[x_{0}, \ldots, x_{n}\right]\right)}{\nu\left({ }_{0}\left[x_{1}, \ldots, x_{n}\right]\right)} \rightrightarrows \frac{e^{\varphi(x)}}{\lambda} \quad \text { as } n \rightarrow \infty
$$

and the case of general $p$ follows from this, as does the second conclusion of the corollary.

Note that we can write the first conclusion of Corollary 1.3 as

$$
\frac{\nu\left({ }_{0}\left[x_{0}, \ldots, x_{n+p-1}\right]\right)}{\nu\left(T^{p}{ }_{0}\left[x_{0}, \ldots, x_{n+p-1}\right]\right)} \rightrightarrows \frac{e^{\left(T_{p} \varphi\right)(x)}}{\lambda^{p}} \quad \text { as } n \rightarrow \infty,
$$


and the second conclusion as

$$
\frac{\nu\left(T^{-p_{0}}\left[w_{0}, \ldots, w_{n-1}\right]\right)}{\left.\nu{ }_{0}\left[w_{0}, \ldots, w_{n-1}\right]\right)} \rightrightarrows \frac{\left(\mathcal{L}_{\varphi}^{p} 1\right)(w)}{\lambda^{p}} \quad \text { as } n \rightarrow \infty .
$$

The following result gives a condition for a probability measure to be an eigenmeasure of a Ruelle operator.

Corollary 1.4. Let $T: X \longrightarrow X$ be a one-sided topologically mixing subshift of finite type and let $\nu \in M(X)$. Then $\mathcal{L}_{\varphi}^{*} \nu=\lambda \nu$ for some $\varphi \in C(X)$ and some $\lambda>0$ if and only if $\nu$ is supported and $\nu\left({ }_{0}\left[x_{0}, \ldots, x_{n}\right]\right) / \nu\left({ }_{0}\left[x_{1}, \ldots, x_{n}\right]\right)$ converges uniformly on $X$ to a function $f: X \longrightarrow(0, \infty)$.

Proof. If $\mathcal{L}_{\varphi}^{*} \nu=\lambda \nu$ for some $\nu \in M(X)$ and some $\lambda>0$, then

$$
\frac{\nu\left({ }_{0}\left[x_{0}, \ldots, x_{n}\right]\right)}{\nu\left({ }_{0}\left[x_{1}, \ldots, x_{n}\right]\right)} \rightrightarrows \frac{e^{\varphi(x)}}{\lambda}
$$

by Corollary 1.3.

If $\nu\left({ }_{0}\left[x_{0}, \ldots, x_{n}\right]\right) / \nu\left({ }_{0}\left[x_{1}, \ldots, x_{n}\right]\right)$ converges uniformly on $X$ to a positive function, then denote the limit by $e^{\varphi(x)}$. We show that $\mathcal{L}_{\varphi}^{*} \nu=\nu$. For any cylinder ${ }_{0}\left[b_{0}, \ldots, b_{t-1}\right]$,

$$
\begin{aligned}
\left(\mathcal{L}_{\varphi}^{*} \nu\right) & \left({ }_{0}\left[b_{0}, \ldots, b_{t-1}\right]\right) \\
& =\int \mathcal{L}_{\varphi \chi_{0}\left[b_{0}, \ldots, b_{t-1}\right]} d \nu=\int_{x \in_{0}\left[b_{1}, \ldots, b_{t-1}\right]} e^{\varphi\left(b_{0} x\right)} d \nu(x) \\
& =\lim _{n \rightarrow \infty} \int_{x \in_{0}\left[b_{1}, \ldots, b_{t-1}\right]} \frac{\nu\left({ }_{0}\left[b_{0}, b_{1}, \ldots, b_{t-1}, x_{t-1}, x_{t}, \ldots, x_{t+n}\right]\right)}{\nu\left({ }_{0}\left[b_{1}, \ldots, b_{t-1}, x_{t-1}, \ldots, x_{t+n}\right]\right)} d \nu(x) \\
& =\lim _{n \rightarrow \infty} \sum_{x_{t-1}, \ldots, x_{t+n}} \nu\left({ }_{0}\left[b_{0}, b_{1}, \ldots, b_{t-1}, x_{t-1}, \ldots, x_{t+n}\right]\right) \\
& =\nu\left({ }_{0}\left[b_{0}, \ldots, b_{t-1}\right]\right) .
\end{aligned}
$$

With Corollary 1.3 in mind, we can characterize when $\varphi \in C(X)$ is a member of $W(X, T)$ in terms of an eigenmeasure $\nu$. If $\mathbb{N}$ is the set of natural numbers, then $\mathrm{BC}(\mathbb{N} \times X)$ denotes the space of bounded continuous real-valued functions on $\mathbb{N} \times X$, equipped with the supremum norm.

TheOrem 1.5. Let $T: X \longrightarrow X$ be a one-sided topologically mixing subshift of finite type and let $\varphi \in C(X)$. Let $\lambda=e^{P(T, \varphi)}$. The following statements are pairwise equivalent.

(i) $\varphi \in W(X, T)$.

(ii) There exists $\tau \in M(X)$ with support $X$ satisfying

$$
\frac{\tau\left({ }_{0}\left[x_{0}, \ldots, x_{p+n-1}\right]\right)}{\tau\left({ }_{0}\left[x_{p}, \ldots, x_{p+n-1}\right]\right)} \frac{\lambda^{p}}{e^{\left(T_{p} \varphi\right)(x)}} \rightrightarrows 1 \quad \text { as } n \rightarrow \infty,
$$

where the convergence is uniform in both $x \in X$ and $p \in \mathbb{N}$. 
(iii) There exists $\nu \in M(X)$ with $\mathcal{L}_{\varphi}^{*} \nu=\lambda \nu$ such that in $\mathrm{BC}(\mathbb{N} \times X)$, the sequence $\left(\psi_{n}\right)$, given by

$$
\psi_{n}(p, x)=\log \left(\frac{\nu\left({ }_{0}\left[x_{0}, \ldots, x_{p+n-1}\right]\right)}{\nu\left({ }_{0}\left[x_{p}, \ldots, x_{p+n-1}\right]\right)}\right) \text {, }
$$

is convergent.

(iv) There exists $\mu \in M(X, T)$ with support $X$ and $\ell \in C(X)$ satisfying

$$
\log \left(\frac{\mu\left(\left[x_{0}, \ldots, x_{n+p-1}\right]\right)}{\mu\left(\left[x_{p}, \ldots, x_{n+p-1}\right]\right)}\right) \rightrightarrows\left(T_{p} \varphi\right)(x)+\ell(x)-\ell\left(T^{p} x\right)-p \log \lambda \quad \text { as } n \rightarrow \infty,
$$

where the convergence is uniform in both $x \in X$ and $p \geqslant 1$.

Proof. The implication (i) $\Rightarrow$ (ii) follows immediately from Theorem 1.2(ii). To show that (ii) $\Rightarrow(\mathrm{i})$, suppose that $\tau \in M(X)$ satisfies (ii). Then for all $\varepsilon>0$, there exists $N_{\varepsilon}$ such that $n \geqslant N_{\varepsilon}$ implies that

$\frac{\tau\left({ }_{0}\left[x_{0}, \ldots, x_{n+p-1}\right]\right)}{\tau\left({ }_{0}\left[x_{p}, \ldots, x_{n+p-1}\right]\right)} \lambda^{p} e^{-\varepsilon}<e^{\left(T_{p} \varphi\right)(x)}<\frac{\tau\left({ }_{0}\left[x_{0}, \ldots, x_{p+n-1}\right]\right)}{\tau\left({ }_{0}\left[x_{p}, \ldots, x_{p+n-1}\right]\right)} \lambda^{p} e^{\varepsilon} \forall p \geqslant 1, \forall x \in X$.

If $x, z \in X$ have $x_{i}=z_{i}$ for $0 \leqslant i \leqslant p+n-1$, then when $n \geqslant N_{\varepsilon}, \quad e^{-2 \varepsilon}<$ $e^{\left(T_{p} \varphi\right)(x)-\left(T_{p} \varphi\right)(z)}<e^{2 \varepsilon}$. Hence $n \geqslant N_{\varepsilon} \Rightarrow \sup _{p} \geqslant 1 \nu_{p+n}\left(T_{p} \varphi\right) \leqslant 2 \varepsilon$. Hence $\varphi \in W(X, T)$.

The implication that (i) $\Rightarrow$ (iii) follows from Theorem 1.2(ii). If (iii) holds, then the limit is $\left(T_{p} \varphi\right)(n)-p \log \lambda$ by Corollary 1.3 , so (ii) holds.

We now show that (i) $\Rightarrow($ iv). When $\varphi \in W(X, T)$, the unique $\nu \in M(X)$ with $\mathcal{L}_{\varphi}^{*} \nu=\lambda \nu$ satisfies (ii), as we saw above. By [11], there exists $h \in C(X), h>0$, with $\mathcal{L}_{\varphi} h=\lambda h$ and $\int h d \nu=1$. The measure $\mu=h \nu \in M(X, T)$, and

$$
e^{-v_{t}(\log h)} \leqslant \frac{\mu\left(\left[x_{0}, \ldots, x_{t-1}\right]\right)}{\nu\left({ }_{0}\left[x_{0}, \ldots, x_{t-1}\right]\right) h(x)} \leqslant e^{v_{t}(\log h)} \quad \forall t \geqslant 1, x \in X .
$$

Hence

$$
\frac{\mu\left(\left[x_{0}, \ldots, x_{n+p-1}\right]\right)}{\mu\left(\left[x_{p}, \ldots, x_{n+p-1}\right]\right)} \frac{\lambda^{p}}{e^{\left(T_{p} \varphi\right)(x)}} \frac{h\left(T^{p} x\right)}{h(x)} \rightrightarrows 1 \quad \text { as } n \rightarrow \infty
$$

uniformly in $p \geqslant 1$ and $x \in X$. Statement (iv) follows with $\ell=\log h$.

If (iv) holds, then, for $\varepsilon>0$, there exists $N_{\varepsilon}$ such that $n \geqslant N_{\varepsilon}$ implies that

$$
e^{-\varepsilon}<\frac{\mu\left(\left[x_{0}, \ldots, x_{n+p-1}\right]\right)}{\mu\left(\left[x_{p}, \ldots, x_{n+p-1}\right]\right)} \frac{\lambda^{p}}{e^{\left(T_{p} \varphi\right)(x)}} \frac{h\left(T^{p} x\right)}{h(x)}<e^{\varepsilon} \quad \forall p \geqslant 1, \forall x \in X,
$$

where $h=e^{\ell}$. If $\left(x_{0}, \ldots, x_{n+p-1}\right)=\left(z_{0}, \ldots, z_{n+p-1}\right)$, then, for $n \geqslant N_{\varepsilon}$,

so

$$
\frac{h\left(T^{p} x\right)}{h\left(T^{p} z\right)} \frac{h(z)}{h(x)} e^{-2 \varepsilon}<e^{\left(T_{p} \varphi\right)(x)-\left(T_{p} \varphi\right)(z)}<\frac{h\left(T^{p} x\right)}{h\left(T^{p} z\right)} \frac{h(z)}{h(x)} e^{2 \varepsilon},
$$

$$
v_{n+p}\left(T_{p} \varphi\right) \leqslant v_{n}(\log h)+v_{n+p}(\log h)+2 \varepsilon \leqslant 2 v_{n}(\log h)+2 \varepsilon \quad \forall p \geqslant 1, \forall n \geqslant 1 .
$$

Hence $\varphi \in W(X, T)$.

Note that when $\varphi \in W(X, T)$, then the eigenmeasure $\nu$ has the property given in (ii). The property in (ii) can be written as

$$
\log \left(\frac{\tau\left({ }_{0}\left[x_{0}, \ldots, x_{n+p-1}\right]\right)}{\tau\left({ }_{0}\left[x_{p}, \ldots, x_{n+p-1}\right]\right)}\right) \rightrightarrows\left(T_{p} \varphi\right)(x)-p \log \lambda \quad \text { as } n \rightarrow \infty,
$$

where the convergence is uniform in both $x \in X$ and $p \geqslant 1$. 
We now characterize, in terms of eigenmeasure, those $\varphi \in C(X)$ which belong to $\operatorname{Bow}(X, T)$.

Definition 1.6. Let $X$ be a one-sided topologically mixing subshift of finite type. Let $\tau \in M(X)$. We say that $\tau$ is approximately multiplicative at coordinate zero if it has support $X$ and there exists $C>1$ with

$$
C^{-1} \leqslant \frac{\tau\left({ }_{0}\left[x_{0}, \ldots, x_{n+p-1}\right]\right)}{\tau\left({ }_{0}\left[x_{0}, \ldots, x_{p-1}\right]\right) \tau\left({ }_{0}\left[x_{p}, \ldots, x_{n+p-1}\right]\right)} \leqslant C \quad \forall n \geqslant 1, p \geqslant 1, x \in X .
$$

If we let $f^{(n)}(x)=\nu\left({ }_{0}\left[x_{0}, \ldots, x_{n-1}\right]\right)$, then the condition becomes

$$
C^{-1} \leqslant \frac{f^{(p+n)}(x)}{f^{(p)}(x) f^{(n)}\left(T^{p} x\right)} \leqslant C .
$$

TheOREM 1.7. Let $T: X \longrightarrow X$ be a one-sided topologically mixing subshift of finite type and let $\varphi \in C(X)$. Let $\lambda=e^{P(T, \varphi)}$. The following statements are pairwise equivalent.

(i) $\varphi \in \operatorname{Bow}(X, T)$.

(ii) There exists $\tau \in M(X)$ with support $X$ and there exists $D>1$ with

$$
D^{-1} \leqslant \tau\left({ }_{0}\left[x_{0}, \ldots, x_{p-1}\right]\right) \frac{\lambda^{p}}{e^{\left(T_{p} \varphi\right)(x)}} \leqslant D \quad \forall p \geqslant 1, \forall x \in X .
$$

(iii) There exists $\nu \in M(X)$ with $\mathcal{L}_{\varphi}^{*} \nu=\lambda \nu$, and $\nu$ is approximately multiplicative at coordinate zero.

Proof. The implication (i) $\Rightarrow$ (ii) follows from Theorem 1.2(i). If (ii) holds and $\left(x_{0}, \ldots, x_{p-1}\right)=\left(z_{0}, \ldots, z_{p-1}\right)$, then

$$
D^{-2} \leqslant e^{\left(T_{p} \varphi\right)(x)-\left(T_{p} \varphi\right)(z)} \leqslant D^{2},
$$

so

$$
v_{p}\left(T_{p} \varphi\right) \leqslant 2 \log D \quad \text { and } \quad \varphi \in \operatorname{Bow}(X, T) .
$$

To show (i) $\Rightarrow$ (iii), we use Theorem 1.2(i) and (ii) to get

$$
\begin{aligned}
\frac{\lambda^{M}}{\lambda^{M} e^{M\|\varphi\|} e^{v_{n+p}\left(T_{p} \varphi\right)+v_{p}\left(T_{p} \varphi\right)}} & \leqslant \frac{\nu\left({ }_{0}\left[x_{0}, \ldots, x_{n+p-1}\right]\right)}{\nu\left({ }_{0}\left[x_{0}, \ldots, x_{p-1}\right]\right) \nu\left({ }_{0}\left[x_{p}, \ldots, x_{n+p-1}\right]\right)} \\
& \leqslant \lambda^{M} e^{M\|\varphi\|} e^{v_{n+p}\left(T_{p} \varphi\right)+v_{p}\left(T_{p} \varphi\right)} .
\end{aligned}
$$

Since $v_{p+n}\left(T_{p} \varphi\right) \leqslant \operatorname{var}_{p}\left(T_{p} \varphi\right)$, we have (i) $\Rightarrow$ (iii). To see that (iii) $\Rightarrow$ (ii), if $C$ is the constant in the definition of $\nu$ being approximately multiplicative at coordinate zero, then from Theorem 1.2(ii) we have

$$
C^{-1} e^{-v_{p+n}\left(T_{p} \varphi\right)} \leqslant \nu\left({ }_{0}\left[x_{0}, \ldots, x_{p-1}\right]\right) \frac{\lambda^{p}}{e^{\left(T_{p} \varphi\right)(x)}} \leqslant C e^{v_{p+n}\left(T_{p} \varphi\right)} \quad \text { for all } n \geqslant 1 .
$$

Let $n \rightarrow \infty$ to give (ii).

The equivalence of (i) and (ii) is well known. Other equivalent conditions for $\varphi \in \operatorname{Bow}(X, T)$ can be found in [14].

As we shall see in Section 3, Theorem 1.7 gives a nice characterization of which $g$-measures correspond to a $g$ with $\log g \in \operatorname{Bow}(X, T)$. 
Corollary 1.8. Let $T: X \longrightarrow X$ be a one-sided topologically mixing subshift of finite type and let $\varphi \in \operatorname{Bow}(X, T)$. The unique equilibrium state $\mu_{\varphi}$ of $\varphi$ is approximately multiplicative at coordinate zero.

Proof. When $\varphi \in \operatorname{Bow}(X, T)$, there is a unique $\nu \in M(X)$ with $\mathcal{L}_{\varphi}^{*} \nu=\lambda \nu[\mathbf{1 4}]$, and $\nu$ is approximately multiplicative at coordinate zero by Theorem 1.7. Also, $\mu_{\varphi}=h \nu$ for some measurable $h: X \longrightarrow[a, b]$ with $0<a<b$, and $\mathcal{L}_{\varphi} h=\lambda h$ and $\int h d \nu=1[\mathbf{1 4}]$. Hence

$$
\begin{aligned}
a \nu\left({ }_{p}\left[x_{0}, \ldots, x_{n-1}\right]\right) \leqslant \mu_{\varphi}\left({ }_{p}\left[x_{0}, \ldots, x_{n-1}\right]\right) \leqslant & b \nu\left({ }_{p}\left[x_{0}, \ldots, x_{n-1}\right]\right) \\
& \forall p \geqslant 1, n \geqslant 1, x \in X,
\end{aligned}
$$

so that $\mu_{\varphi}$ is approximately multiplicative at coordinate zero.

Since $\mu_{\varphi}$ is $T$-invariant, we can write the approximately multiplicative at coordinate zero condition for $\mu_{\varphi}$ as follows. There exists $D>1$ with

$$
D^{-1} \leqslant \frac{\mu_{\varphi}\left[0\left(x_{0}, \ldots, x_{n+p-1}\right)\right]}{\mu_{\varphi}\left({ }_{0}\left[x_{0}, \ldots, x_{p-1}\right]\right) \mu_{\varphi}\left({ }_{p}\left[x_{p}, \ldots, x_{n+p-1}\right]\right)} \leqslant D \quad \forall p \geqslant 1, n \geqslant 1, x \in X .
$$

We use this condition in the next definition.

Definition 1.9. Let $T: X \longrightarrow X$ be a one-sided topologically mixing subshift of finite type and let $\tau \in M(X)$. We say that $\tau$ has approximate product structure if it has support $X$ and if there exists $C>1$ with

$$
C^{-1} \leqslant \frac{\tau\left({ }_{0}\left[x_{0}, \ldots, x_{n+p-1}\right]\right)}{\tau\left({ }_{0}\left[x_{0}, \ldots, x_{p-1}\right]\right) \tau\left({ }_{p}\left[x_{p}, \ldots, x_{n+p-1}\right]\right)} \leqslant C \quad \forall n \geqslant 1, p \geqslant 1, x \in X .
$$

If $\tau \in M(X, T)$, then clearly $\tau$ is approximately multplicative at coordinate zero if and only if $\tau$ has approximate product structure. To investigate the relationship between the two conditions when $\tau$ is an eigenmeasure $\nu$ for $\mathcal{L}_{\varphi}$, we can use the following deduction from Theorem 1.2(iii).

Proposition 1.10. Let $T: X \longrightarrow X$ be a one-sided topologically mixing subshift of finite type and let $\varphi \in C(X)$. Let $\nu \in M(X)$ satisfy $\mathcal{L}_{\varphi}^{*} \nu=\lambda \nu$ with $\lambda=e^{P(T, \varphi)}$. Let $D \geqslant 1$, and let $p \in \mathbb{N}$. Then

if and only if

$$
D^{-1} \leqslant \frac{\left(\mathcal{L}_{\varphi}^{p} 1(x)\right)}{\lambda^{p}} \leqslant D \quad \forall x \in X
$$

$$
D^{-1} \leqslant \frac{\nu\left({ }_{p}\left[z_{0}, \ldots, z_{n-1}\right]\right)}{\nu\left({ }_{0}\left[z_{0}, \ldots, z_{n-1}\right]\right)} \leqslant D \quad \forall n \geqslant 1, z \in X .
$$

Proof. By Theorem 1.2(iii),

$$
\nu\left({ }_{p}\left[z_{0}, \ldots, z_{n-1}\right]\right)=\int_{w \in_{0}\left[z_{0}, \ldots, z_{n-1}\right]} \frac{\left(\mathcal{L}_{\varphi}^{p} 1\right)}{\lambda^{p}}(w) d \nu(w) .
$$

Clearly the first statement of the proposition implies the second statement. Now suppose that the second statement holds and let

$$
U=\left\{x \in X \mid \frac{\left(\mathcal{L}_{\varphi}^{p} 1\right)(x)}{\lambda^{p}}(x)>D\right\} \text {. }
$$


Then $U$ is open. Suppose that $U \neq \varnothing$. For $z \in U$, choose some $n$ with ${ }_{0}\left[z_{0}, \ldots, z_{n-1}\right] \subset U$. Then, by $(*), \nu\left({ }_{p}\left[z_{0}, \ldots, z_{n-1}\right]\right)>D \nu\left({ }_{0}\left(\left[z_{0}, \ldots, z_{n-1}\right]\right)\right.$, which contradicts the assumption. Hence $U=\varnothing$, so $\left(\mathcal{L}_{\varphi}^{p} 1\right)(x) / \lambda^{p} \leqslant D$ for all $x \in X$. Similarly, we get $D^{-1} \leqslant\left(\mathcal{L}_{\varphi}^{p} 1\right)(x) / \lambda^{p}$ for all $x \in X$.

Corollary 1.11. Let $T: X \longrightarrow X$ be a one-sided topologically mixing subshift of finite type and let $\varphi \in C(X)$. Let $\nu \in M(X)$ satisfy $\mathcal{L}_{\varphi}^{*} \nu=\lambda \nu$ with $\lambda=e^{P(T, \varphi)}$. Suppose that there exists $D \geqslant 1$ with

$$
D^{-1} \leqslant \frac{\left(\mathcal{L}_{\varphi}^{p} 1\right)(x)}{\lambda^{p}} \leqslant D \quad \forall p \geqslant 1, \forall x \in X .
$$

Then $\nu$ is approximately multiplicative at coordinate zero if and only if $\nu$ has approximate product structure.

Corollary 1.12. Let $T: X \longrightarrow X$ be a one-sided topologically mixing subshift of finite type and let $\varphi \in C(X)$. Let $\lambda=e^{P(T, \varphi)}$. Then $\varphi \in \operatorname{Bow}(X, T)$ if and only if both of the following statements hold.

(i) There exists $B>1$ with $B^{-1} \leqslant\left(\mathcal{L}_{\varphi}^{p} 1\right)(x) / \lambda^{p} \leqslant B$ for all $p \geqslant 1, x \in X$.

(ii) There exists $\nu \in M(X)$ with $\mathcal{L}_{\varphi}^{*} \nu=\lambda \nu$ and $\nu$ has approximate product structure.

Proof. If $\varphi \in \operatorname{Bow}(X, T)$, then (i) holds by [14, p. 337], and (ii) holds by Theorem 1.7 and Corollary 1.11. If (i) and (ii) hold, then, by Corollary 1.11, $\nu$ is approximately multiplicative at coordinate zero and hence $\varphi \in \operatorname{Bow}(X, T)$ by Theorem 1.7.

The following lemma is well known.

Lemma 1.13. Let $T: X \longrightarrow X$ be a one-sided topologically mixing subshift of finite type and let $\varphi \in C(X)$ and $\lambda=e^{P(T, \varphi)}$. Suppose that $\nu \in M(X)$ satisfies $\mathcal{L}_{\varphi}^{*} \nu=\lambda \nu$, and suppose that there is a measurable $h: X \longrightarrow[a, b]$ with $0<a \leqslant b$ and $\mathcal{L}_{\varphi} h=\lambda h$. Suppose that $h$ is normalized so that $\int h d \nu=1$. Then $\mu=h \nu$ is an equilibrium state for $\varphi$.

Proof. We have $\mu \in M(X, T)$ since, for $f \in C(X)$,

$$
\int f \circ T d \mu=\int f \circ T h d \nu=\lambda^{-1} \int \mathcal{L}_{\varphi}(f \circ T h) d \nu=\lambda^{-1} \int f \mathcal{L}_{\varphi}(h) d \nu=\int f h d \nu=\int f d \mu .
$$

To see that $\mu$ is an equilibrium state, we use Theorem 1.2(i) to get

$$
\begin{aligned}
-\frac{1}{p} \log \left(\lambda^{M} e^{M\|\varphi\|}\right)-\frac{v_{p}\left(T_{p} \varphi\right)}{p} & \leqslant-\frac{1}{p} \log \nu\left({ }_{0}\left[x_{0}, \ldots, x_{p-1}\right]\right)+\frac{1}{p}\left(T_{p} \varphi\right)(x)-\log \lambda \\
& \leqslant \frac{1}{p} \log \left(\frac{k^{M} e^{M\|\varphi\|}}{\lambda^{M}}\right)+\frac{v_{p}\left(T_{p} \varphi\right)}{p} .
\end{aligned}
$$

Since $a \nu\left({ }_{0}\left[x_{0}, \ldots, x_{p-1}\right]\right) \leqslant \mu\left({ }_{0}\left[x_{0}, \ldots, x_{p-1}\right]\right) \leqslant b \nu\left({ }_{0}\left[x_{0}, \ldots, x_{p-1}\right]\right)$ and

$$
v_{p}\left(T_{p} \varphi\right) \leqslant \sum_{i=1}^{p} v_{i}(\varphi)
$$


we have

$$
-\frac{1}{p} \log \mu\left({ }_{0}\left[x_{0}, \ldots, x_{p-1}\right]\right)+\frac{1}{p}\left(T_{p} \varphi\right)(x) \Rightarrow \log \lambda=P(T, \varphi) .
$$

Integrating via $\mu$ gives

$$
\frac{1}{p} H\left(\bigvee_{i=0}^{p-1} T^{-i} \xi\right)+\int \varphi d \mu \longrightarrow P(T, \varphi),
$$

where $\xi$ is the partition into states at coordinate zero. Since $\xi$ is a one-sided generator, we have $h_{\mu}(T)+\int \varphi d \mu=P(T, \varphi)$.

There is another characterization of $\varphi \in \operatorname{Bow}(X, T)$.

TheOREM 1.14. Let $T: X \longrightarrow X$ be a one-sided topologically mixing subshift of finite type and let $\varphi \in C(X)$ and $\lambda=e^{P(T, \varphi)}$. Then $\varphi \in \operatorname{Bow}(X, T)$ if and only if all of the following three statements hold.

(i) $\varphi$ has a unique equilibrium state $\mu_{\varphi}$.

(ii) $\mu_{\varphi}$ has approximate product structure.

(iii) There exists $B>1$ with $B^{-1} \leqslant\left(\mathcal{L}_{\varphi}^{p} 1\right)(x) / \lambda^{p} \leqslant B$ for all $p \geqslant 1, x \in X$.

Proof. If $\varphi \in \operatorname{Bow}(X, T)$, (i) and (iii) hold by [14], and (ii) holds by Corollary 1.8. Now assume that the three conditions hold. Let $\nu \in M(X)$ satisfy $\mathcal{L}_{\varphi}^{*} \nu=\lambda \nu$. By (iii), there is a measurable $h: X \longrightarrow[a, b]$ with $0<a<b, \int h d \nu=1$, and $\mathcal{L}_{\varphi} h=\lambda h$ [14, p. 341]. Then, by Lemma 1.13, $\mu=h \nu$ is $T$-invariant and is an equilibrium state for $\varphi$. By (i), $\mu_{\varphi}=h \nu$. By (ii), $\mu_{\varphi}$ is approximately multiplicative at coordinate zero, so $\nu$ is also. Hence $\varphi \in \operatorname{Bow}(X, T)$ by Theorem 1.7 .

\section{The weak Bernoulli property}

In this section, we show that if $\varphi \in \operatorname{Bow}(X, T)$, then the natural extension of $T$ with respect to the unique equilibrium state $\mu_{\varphi}$ of $\varphi$ is a Bernoulli shift. We shall also show that when $S: \hat{X} \longrightarrow \hat{X}$ is a two-sided topologically mixing subshift of finite type and $\hat{\varphi} \in \operatorname{Bow}(\hat{X}, S)$, then $\left(S, \hat{\mu}_{\hat{\varphi}}\right)$ is isomorphic to a Bernoulli shift where $\hat{\mu}_{\hat{\varphi}}$ is the unique equilibrium state of $\hat{\varphi}$.

When $\hat{\varphi} \in \operatorname{Bow}(\hat{X}, S)$, then Bowen $[\mathbf{2}]$ showed that there exists $C>1$ with

$$
C^{-1} \leqslant \frac{\hat{\mu}_{\hat{\varphi}}\left(\left[x_{0}, \ldots, x_{n-1}\right]\right)}{e^{\left(S_{n} \hat{\varphi}\right)(x)-n P(S, \hat{\varphi})}} \leqslant C \quad \forall x \in \hat{X}, \forall n \geqslant 1 .
$$

It readily follows that $\hat{\mu}_{\hat{\varphi}}$ has approximate product structure (or rather that $\hat{\mu} \circ \pi^{-1}$ does, where $\pi: \hat{X} \longrightarrow X$ is the natural projection). The definition of approximate product structure also makes sense for a measure on the two-sided shift space $\hat{X}$.

Whereas every $\hat{\varphi} \in W(\hat{X}, S)$ is cohomologous in $C(\hat{X})$ to some $\varphi \circ \pi$ with $\varphi \in W(X, T)[\mathbf{1}]$, there are examples of $\hat{\varphi} \in \operatorname{Bow}(\hat{X}, S)$ which are not cohomologous in $C(\hat{X})$ to a one-sided function $[\mathbf{1 0}]$.

For every $\mu \in M(X, T)$, there is a unique $\hat{\mu} \in M(\hat{X}, S)$ with $\hat{\mu} \circ \pi^{-1}=\mu$. We have $\hat{\mu}\left({ }_{p}\left[b_{0}, \ldots, b_{n-1}\right]\right)=\mu\left({ }_{q}\left[b_{0}, \ldots, b_{n-1}\right]\right)$, for all $p \in Z, q \geqslant 0$. This gives a bijection between $M(X, T)$ and $M(\hat{X}, S)$. 
ThEOREM 2.1. Let $T: X \longrightarrow T$ be a one-sided topologically mixing subshift of finite type with transition matrix $A$. Let $\mu \in M(X, T)$ have approximate product structure. If $A^{M}>0$, then there exists $D>1$ such that for all $n \geqslant 2 M$, all $p, q \geqslant 1$, and all allowable cylinders ${ }_{0}\left[x_{0}, \ldots, x_{p-1}\right],{ }_{0}\left[y_{0}, \ldots, y_{q-1}\right]$, we have

$$
D^{-1} \leqslant \frac{\mu\left({ }_{0}\left[x_{0}, \ldots, x_{p-1}\right] \cap T^{-(p+n)}{ }_{0}\left[y_{0}, \ldots, y_{q-1}\right]\right)}{\mu\left(\left[x_{0}, \ldots, x_{p-1}\right]\right) \mu\left(\left[y_{0}, \ldots, y_{q-1}\right]\right)} \leqslant D .
$$

Proof. Let $C$ be the constant in the approximate product structure condition for $\mu$. Let $\left[x_{0}, \ldots, x_{p-1}\right]$ and $\left[y_{0}, \ldots, y_{q-1}\right]$ be given. Let $n \geqslant 2 M$. Since $A^{M}>0$, there is a point $w \in X$ of the form $w=\left(x_{0}, \ldots, x_{p-1}, w_{p}, \ldots, w_{p+n-1}, y_{0}, y_{1}, \ldots\right)$. Moreover, for any allowable choice of the cylinder $\left[w_{p+M-1}, \ldots, w_{p+n-M}\right]$, there is such a point. Since

$$
\begin{aligned}
& C^{-4} \leqslant \\
& \frac{\mu\left[x_{0}, \ldots, x_{p-1}, w_{p}, \ldots, w_{p+n-1}, y_{0}, \ldots, y_{q-1}\right]}{\mu\left[x_{0}, \ldots, x_{p-1}\right] \mu\left[w_{p}, \ldots, w_{p+M-2}\right] \mu\left[w_{p+M-1}, \ldots, w_{p+n-M}\right] \mu\left[w_{p+n-M+1}, \ldots, w_{p+n-1}\right] \mu\left[y_{0}, \ldots, y_{q-1}\right]} \\
& \quad \leqslant C^{4},
\end{aligned}
$$

we have

$$
C^{-4} d^{2} \leqslant \frac{\mu\left({ }_{0}\left[x_{0}, \ldots, x_{p-1}\right] \cap T^{-(p+n)}{ }_{0}\left[y_{0}, \ldots, y_{q-1}\right]\right)}{\mu\left({ }_{0}\left[x_{0}, \ldots, x_{p-1}\right]\right) \mu\left({ }_{0}\left[y_{0}, \ldots, y_{q-1}\right]\right)} \leqslant C^{4},
$$

where $d$ is the minimal $\mu$-measure of a cylinder of length $M-1$.

Therefore, put $D=C^{4} d^{-2}$.

If we consider the corresponding two-sided measure $\hat{\mu}$, then the conclusion of Theorem 2.1 can be written as

$$
D^{-1} \leqslant \frac{\hat{\mu}\left({ }_{-p}\left[x_{-p}, \ldots, x_{-1}\right] \cap S^{-n_{0}}\left[y_{0}, \ldots, y_{q-1}\right]\right)}{\hat{\mu}\left(\left[x_{-p}, \ldots, x_{-1}\right]\right) \hat{\mu}\left(\left[y_{0}, \ldots, y_{q-1}\right]\right)} \leqslant D
$$

for all $n \geqslant 2 M$ and all cylinders ${ }_{-p}\left[x_{-p}, \ldots, x_{-1}\right],{ }_{0}\left[y_{0}, \ldots, y_{q-1}\right]$ with $p, q \geqslant 1$.

By the usual approximation arguments, one can readily get

$$
D^{-1} \leqslant \frac{\hat{\mu}\left(B_{1} \cap S^{-n} B_{2}\right)}{\hat{\mu}\left(B_{1}\right) \hat{\mu}\left(B_{2}\right)} \leqslant D
$$

whenever $n \geqslant 2 M, B_{1} \in \mathcal{B}_{-\infty}^{-1}, B_{2} \in \mathcal{B}_{0}^{\infty}$ and $\hat{\mu}\left(B_{1}\right) \hat{\mu}\left(B_{2}\right)>0$. Here $\mathcal{B}_{-\infty}^{-1}$ is the $\sigma$-algebra generated by all cylinders ${ }_{-p}\left[x_{-p}, \ldots, x_{-1}\right]_{-1}$ with $p \geqslant 1$, and $\mathcal{B}_{0}^{\infty}$ is the $\sigma$-algebra generated by all cylinders $0\left[y_{0}, \ldots, y_{q-1}\right]_{q-1}$ with $q \geqslant 1$.

We shall use the following result.

Corollary 2.2. Let $S: \hat{X} \longrightarrow \hat{X}$ be a one-sided topologically mixing subshift of finite type and let $\hat{\mu} \in M(\hat{X}, S)$ be of approximate product type. Then $S$ is strongly mixing with respect to $\hat{\mu}$.

Proof. This follows from a result of Ornstein $[\mathbf{9}]$ if we can show that $S^{i}$ is ergodic for each $i \geqslant 1$ and there is a constant $d$ with

$$
\limsup _{n \rightarrow \infty} \hat{\mu}\left(B_{1} \cap S^{-n} B_{2}\right) \leqslant d \hat{\mu}\left(B_{1}\right) \hat{\mu}\left(B_{2}\right) \quad \forall B_{1}, B_{2} \in \mathcal{B} .
$$

From $(\dagger)$, we get

$$
\limsup _{n \rightarrow \infty} \hat{\mu}\left(B_{1} \cap S^{-n} B_{2}\right) \leqslant D \hat{\mu}\left(B_{1}\right) \hat{\mu}\left(B_{2}\right)
$$


whenever $B_{1} \in \mathcal{B}_{-\infty}^{\ell}$ and $B_{2} \in \mathcal{B}_{-j}^{\infty}, \ell, j>0$, so an approximation argument gives the same inequality whenever $B_{1}, B_{2} \in \mathcal{B}$.

Similarly,

$$
\liminf _{n \rightarrow \infty} \hat{\mu}\left(B_{1} \cap S^{-n} B_{2}\right) \geqslant D^{-1} \hat{\mu}\left(B_{1}\right) \hat{\mu}\left(B_{2}\right)
$$

whenever $B_{1}, B_{2} \in \mathcal{B}$. This latter inequality gives the total ergodicity required for Ornstein's result, since, if $S^{i} B_{2}=B_{2}$, then

$$
\begin{gathered}
0=\hat{\mu}\left(\left(\hat{X} \backslash B_{2}\right) \cap B_{2}\right) \geqslant D^{-1} \hat{\mu}\left(\hat{X} \backslash B_{2}\right) \hat{\mu}\left(B_{2}\right), \\
\text { so either } \hat{\mu}\left(B_{2}\right) \text { or } \hat{\mu}\left(\hat{X} \backslash B_{2}\right) \text { is } 0 .
\end{gathered}
$$

We now use results of Bradley to show that having approximate product structure implies the weak Bernoulli property.

THEOREM 2.3. Let $S: \hat{X} \longrightarrow \hat{X}$ be a two-sided topologically mixing subshift of finite type, and let $\hat{\mu} \in M(\hat{X}, S)$ have approximate product structure. Then for all $\varepsilon>0$, there exists $N$ so that $n \geqslant N$ implies that

$$
e^{-\varepsilon} \hat{\mu}\left(B_{1}\right) \hat{\mu}\left(B_{2}\right) \leqslant \hat{\mu}\left(B_{1} \cap S^{-n} B_{2}\right) \leqslant e^{\varepsilon} \hat{\mu}\left(B_{1}\right) \hat{\mu}\left(B_{2}\right)
$$

whenever $B_{1} \in \mathcal{B}_{-\infty}^{-1}$ and $B_{2} \in \mathcal{B}_{0}^{\infty}$. Hence the natural partition into states at coordinate zero is a weak Bernoulli partition for $S$, so $(S, \hat{\mu})$ is isomorphic to a Bernoulli shift.

Proof. From Theorem 2.1, the inequalities $(\dagger)$ hold. Since, by Corollary 2.2, $(S, \hat{\mu})$ is strongly mixing, then, by a result of Bradley [3], if

$$
\begin{aligned}
& \psi_{n}^{*}=\sup \left\{\frac{\hat{\mu}\left(B_{1} \cap S^{-n} B_{2}\right)}{\hat{\mu}\left(B_{1}\right) \hat{\mu}\left(B_{2}\right)} \mid B_{1} \in \mathcal{B}_{-\infty}^{-1}, B_{2} \in \mathcal{B}_{0}^{\infty}, \hat{\mu}\left(B_{1}\right) \hat{\mu}\left(B_{2}\right)>0\right\} \\
& \psi_{n}^{\prime}=\inf \left\{\frac{\hat{\mu}\left(B_{1} \cap S^{-n} B_{2}\right)}{\hat{\mu}\left(B_{1}\right) \hat{\mu}\left(B_{2}\right)} \mid B_{1} \in \mathcal{B}_{-\infty}^{-1}, B_{2} \in \mathcal{B}_{0}^{\infty}, \hat{\mu}\left(B_{1}\right) \hat{\mu}\left(B_{2}\right)>0\right\},
\end{aligned}
$$

then (i) either $\psi_{n}^{*} \rightarrow 1$ as $n \rightarrow \infty$ or $\psi_{n}^{*}=\infty$ for all $n$, (ii) either $\psi_{n}^{\prime} \rightarrow 1$ as $n \rightarrow \infty$ or $\psi_{n}^{\prime}=0$ for all $n$. Since inequalities ( $\dagger$ ) hold, we have $\psi_{n}^{*} \leqslant D$ and $D^{-1} \leqslant \psi_{n}^{\prime}$, so $\psi_{n}^{*} \rightarrow 1$ and $\psi_{n}^{\prime} \rightarrow 1$. This gives the condition in the statement of the theorem. The isomorphism result is due to Friedman and Ornstein [5].

We state the following results.

Theorem 2.4. Let $T: X \longrightarrow X$ be a one-sided, and let $S: \hat{X} \longrightarrow \hat{X}$ be the corresponding two-sided topologically mixing subshift of finite type.

(i) If $\varphi \in \operatorname{Bow}(X, T)$ and $\mu_{\varphi}$ is its unique equlibrium state, then the natural extension of $\left(T, \mu_{\varphi}\right)$ is isomorphic to a Bernoulli shift.

(ii) If $\hat{\varphi} \in \operatorname{Bow}(\hat{X}, S)$ and $\hat{\mu}_{\hat{\varphi}}$ is its unique equilibrium state, then $\left(S, \hat{\mu}_{\varphi}\right)$ is isomorphic to a Bernoulli shift.

Proof. (i) By Corollary 1.8, $\mu_{\varphi}$ has approximate product structure, so $\hat{\mu}_{\varphi}$ has approximate product structure. Now Theorem 2.3 gives the result.

(ii) Bowen $[2]$ showed that $\hat{\mu}_{\hat{\varphi}}$ has the property that there exists $C>1$ with

$$
C^{-1} \leqslant \frac{\left.\hat{\mu}_{\hat{\varphi}}\left[x_{0}, \ldots, x_{n-1}\right]\right) \varphi}{e^{\left(S_{n} \hat{\varphi}\right)(x)-n P(S, \hat{\varphi})}} \leqslant C \quad \forall n \geqslant 1, x \in \hat{X} .
$$


Hence $\hat{\mu}_{\hat{\varphi}}$ has approximate product structure, so the result follows by Theorem 2.3.

\section{3. $g$-measures}

We interpret the results in Sections 1 and 2 for the case of $g$-measures. If $T: X \longrightarrow X$ is a one-sided topologically mixing subshift of finite type, let $\mathcal{G}(X, T)$, or $\mathcal{G}$, denote the set

$$
\left\{g \in C(X) \mid g(x)>0 \forall x \in X \text { and } \sum_{y \in T^{-1} x} g(y)=1 \forall x \in X\right\} .
$$

If $g \in \mathcal{G}$, we can consider $\mathcal{L}_{\log g}$, and $\mu \in M(X)$ is called a $g$-measure if $\mathcal{L}_{\log g}^{*} \mu=\mu$ [7]. Such a measure always belongs to $M(X, T)$. The condition can be formulated in several ways. For example, one can show that $\mu \in M(X, T)$ is a $g$ measure if and only if $\mu$ is an equilibrium state of $\log g([\mathbf{8}]$, see also [11]). Since $P(T, \log g)=0$ for $g \in \mathcal{G}$, this condition becomes $h_{\mu}(T)+\int \log g d \mu=0$. Let $\mathcal{M}(X, T)=\{\mu \in M(X, T) \mid \mu$ is a $g$-measure for some $g \in \mathcal{G}(X, T)\}$.

The following results are special cases of the results in Sections 1 and 2, obtained by considering $\varphi$ of the form $\log g$ with $g \in \mathcal{G}(X, T)$. Again, $k$ is the number of symbols used for the subshift of finite type, and $M$ is a natural number with $A^{M}>0$.

Theorem 3.1. Let $T: X \longrightarrow X$ be a one-sided topologically mixing subshift of finite type and let $g \in \mathcal{G}$. Let $\mu$ be a $g$-measure. Then $\mu$ has support $X$ and each of the following holds.

(i) For all $p \geqslant 1, x \in X$,

$$
(\inf g)^{M} e^{-v_{p}\left(T_{p} \log g\right)} \leqslant \frac{\mu\left[x_{0}, \ldots, x_{p-1}\right]}{g(x) g(T x) \ldots g\left(T^{p-1} x\right)} \leqslant k^{M} e^{v_{p}\left(T_{p} \log g\right)} .
$$

(ii) For all $n, p \leqslant 1, x \in X$,

$$
e^{-v_{n+p}\left(T_{p} \log g\right)} \leqslant \frac{\mu\left(\left[x_{0}, \ldots, x_{p+n-1}\right]\right)}{\mu\left(\left[x_{p}, \ldots, x_{p+n}\right]\right) g(x) g(T x) \ldots g\left(T^{p-1} x\right)} \leqslant e^{v_{n+p}\left(T_{p} \log g\right)} .
$$

Corollary 3.2. For $T: X \longrightarrow X, g \in \mathcal{G}$ and $\mu \in M(X, T)$ as in Theorem 3.1, we have for each fixed $p \geqslant 1$,

$$
\frac{\mu\left(\left[x_{0}, \ldots, x_{p+n-1}\right]\right)}{\mu\left(\left[x_{p}, \ldots, x_{p+n-1}\right]\right)} \rightrightarrows g(x) g(T x) \ldots g\left(T^{p-1} x\right) \quad \text { as } n \rightarrow \infty .
$$

Corollary 3.3. Let $T: X \longrightarrow X$ be a one-sided topologically mixing subshift of finite type and let $\mu \in M(X, T)$. Then $\mu$ is a $g$-measure for some $g \in \mathcal{G}$ if and only if $\mu$ has support $X$ and $\mu\left(\left[x_{0}, \ldots, x_{n-1}\right]\right) / \mu\left(\left[x_{1}, \ldots, x_{n-1}\right]\right)$ converges uniformly on $X$ as $n \rightarrow \infty$ to a function $f: X \longrightarrow(0, \infty)$.

With Corollary 3.2 in mind, we can characterize those $g$ with $\log g \in W(X, T)$ as follows.

Theorem 3.4. Let $T: X \longrightarrow X$ be a one-sided topologically mixing subshift of finite type and let $g \in \mathcal{G}$. The following statements are pairwise equivalent.

(i) $\log g \in W(X, T)$. 
(ii) There exists $\mu \in M(X, T)$, with support $X$, satisfying

$$
\log \left(\frac{\mu\left(\left[x_{0}, \ldots, x_{p+n-1}\right]\right)}{\mu\left(\left[x_{p}, \ldots, x_{p+n-1}\right]\right)}\right) \rightrightarrows\left(T_{p}(\log g)\right)(x) \quad \text { as } n \rightarrow \infty,
$$

where the convergence is uniform in both $x \in X$ and $p \geqslant 1$.

(iii) There exists a $g$-measure $\mu$ such that in $\operatorname{BC}(\mathbb{N} \times X)$, the sequence $\left(\psi_{n}\right)$, given by

$$
\psi_{n}(p, x)=\log \left(\frac{\mu\left(\left[x_{0}, \ldots, x_{p+n-1}\right]\right)}{\mu\left(\left[x_{p}, \ldots, x_{p+n-1}\right]\right)}\right),
$$

is convergent.

Note that the unique $g$-measure $\mu$, when $\log g \in W(X, T)$, satisfies the condition in (ii).

We shall use Theorem 3.4 later in an application. The following result characterizes those $g$ with $\log g \in \operatorname{Bow}(X, T)$.

Theorem 3.5. Let $T: X \longrightarrow X$ be a one-sided topologically mixing subshift of finite type and let $g \in \mathcal{G}(X, T)$. Then $\log g \in \operatorname{Bow}(X, T)$ if and only if there is a $g$-measure which has approximate product structure. When $\log g \in B(X, T)$, there is a unique $g$-measure $\mu$ and the coordinate zero partition is weak Bernoulli for $\mu$ so that the natural extension of $(T, \mu)$ is isomorphic to a Bernoulli shift.

Proof. Since a $g$-measure is exactly an eigenmeasure for $\mathcal{L}_{\log g}^{*}$ and is $T$-invariant, the first statement follows from Theorem 1.7. Let $\log g \in \operatorname{Bow}(X, T)$. By $[\mathbf{1 4}$, Theorem 3.2], there is a unique $g$-measure, and the Bernoulli properties follow from Theorem 2.4.

Notice that when $\mu$ is $g$-measure, then $\mu$ has approximate product structure if and only if $\log g \in \operatorname{Bow}(X, T)$.

We now consider the question of whether the 'reverse' of a $g$-measure is also a $g$-measure. Let $T: X \longrightarrow X$, where $X=X_{A}$, be a one-sided topologically mixing subshift of finite type, and let $S: \hat{X} \longrightarrow \hat{X}$ be the corresponding twosided topologically mixing subshift of finite type. Let $\pi: \hat{X} \longrightarrow X$ be the natural projection given by $\pi\left\{x_{n}\right\}_{-\infty}^{\infty}=\left\{x_{n}\right\}_{0}^{\infty}$. Then $\pi S=T \pi$, and there is a natural bijection $M(\hat{X}, S) \longrightarrow M(X, T)$ given by $\hat{\mu} \longrightarrow \hat{\mu} \circ \pi^{-1}$. We denote $\hat{\mu} \circ \pi^{-1}$ by $\hat{\mu}_{+}$.

The other one-sided space $X_{-}=\left\{\left\{x_{n}\right\}_{-\infty}^{0} \mid \exists x_{i}\right.$ for $i \geqslant 1$ with $\left.\left\{x_{n}\right\}_{-\infty}^{\infty} \in \hat{X}\right\}$ together with the shift $T_{-}: X_{-} \longrightarrow X_{-}$, given by $T_{-}\left(\left(\ldots, x_{-2}, x_{-1}, x_{0}\right)\right)=\left(\ldots, x_{-2}\right.$, $\left.x_{-1}\right)$, can be considered as the one-sided shift on the space $X_{A^{\mathrm{t}}}$, where $A^{\mathrm{t}}$ is the transpose of the matrix $A$. Let $\pi: \hat{X} \longrightarrow X_{-}$be given by $\pi_{-}\left\{x_{n}\right\}_{-\infty}^{\infty}=\left\{x_{n}\right\}_{-\infty}^{0}$ and then $\pi S^{-1}=T_{-} \pi_{-}$. Since $M\left(\hat{X}, S^{-1}\right)=M(\hat{X}, S)$, we have a natural bijection $M(\hat{X}, S) \longrightarrow M\left(X_{-}, T_{-}\right)$given by $\hat{\mu} \longrightarrow \hat{\mu} \circ \pi_{-}^{-1} \equiv \hat{\mu}_{-}$, so that $\hat{\mu}_{+} \longrightarrow \hat{\mu}_{-}$gives a natural bijection $M(X, T) \longrightarrow M\left(X_{-}, T_{-}\right)$, For an allowed cylinder $\left[i_{1}, \ldots, i_{r}\right]$ in $\hat{X}$, we have $\hat{\mu}_{+}\left({ }_{s}\left[i_{1}, \ldots, i_{r}\right]\right)=\mu_{-}\left(\left[i_{1}, \ldots, i_{r}\right]_{t}\right)$ for all $s \geqslant 0, t \leqslant 0$. Clearly $\hat{\mu}_{+}$ has support $X$ if and only if $\hat{\mu}_{-}$has support $X_{-}$. We can define what it means for $\hat{\mu}_{-}$to have approximate product structure by considering the natural conjugacy $X_{-} \longrightarrow X_{A^{\mathrm{t}}}$, given by $\left(\ldots, x_{-2}, x_{-1}, x_{0}\right) \longrightarrow\left(x_{0}, x_{-1}, x_{-2}, \ldots\right)$, of $T_{-}$to the topologically mixing subshift of finite type on $X_{A^{\mathrm{t}}}$. Then $\hat{\mu}_{-}$has approximate product structure if and only if $\hat{\mu}_{+}$has approximate product structure if and only if $\hat{\mu}$ has approximate product structure. 
Let $\mathcal{G}_{+}$denote $\mathcal{G}(X, T)$, the space of all positive $g$-functions for $T$, and let $\mathcal{G}_{-}$ denote $\mathcal{G}\left(X_{-}, T_{-}\right)$, the space of all positive $g$-functions for $T_{-}$. Hence

$$
\mathcal{G}_{-}=\left\{g \in C\left(X_{-}\right) \mid g(z)>0 \forall z \in X_{-} \text {and } \sum_{w \in T_{-}^{-1} z} g(w)=1 \forall z \in X_{-}\right\} .
$$

If $\mathcal{M}_{+}$denotes $\mathcal{M}(X, T)$ and $\mathcal{M}_{-}$denotes $\mathcal{M}\left(X_{-}, T_{-}\right)$, then the map $\hat{\mu}_{+} \longrightarrow \hat{\mu}_{-}$ need not map $\mathcal{M}_{+}$into $\mathcal{M}_{-}$. Kalikow constructed examples to show this when $X$ is the full shift space on two symbols [6]. One can construct a family of such examples, inspired by Kalikow, as follows.

Let $X=\prod_{0}^{\infty}\{0,1\}$ be the space of all sequences $\left(x_{0}, x_{1}, \ldots\right)$ with each $x_{n} \in\{0,1\}$. Let $\left\{d_{n}\right\}_{n=0}^{\infty}$ be such that $d_{n} \in[0,1)$ for all $n \geqslant 0, d_{n} \rightarrow 0$ as $n \rightarrow \infty$, and $\sum_{n=0}^{\infty}\left(d_{n} /\left(1+d_{n}\right)\right)=\infty$. Such a sequence is given by $d_{n}=1 /(n+1)$. Define $g: X \longrightarrow(0,1)$ as follows. For $k \geqslant 0, \ell \geqslant 0$, put

$$
\begin{aligned}
& g\left(000^{k} 1^{\ell} 101 \ldots\right)=\frac{1}{2}\left(1-d_{k+\ell}\right), \\
& g\left(100^{k} 1^{\ell} 101 \ldots\right)=\frac{1}{2}\left(1+d_{k+\ell}\right), \\
& g\left(000^{k} 1^{\ell} 100 \ldots\right)=\frac{1}{2}\left(1+d_{k+\ell}\right), \\
& g\left(100^{k} 1^{\ell} 100 \ldots\right)=\frac{1}{2}\left(1-d_{k+\ell}\right),
\end{aligned}
$$

and $g(x)=\frac{1}{2}$ at all other points. The value of $g$ depends on the first occurrence of cylinder [10] in $\left(x_{2}, x_{3}, \ldots\right)$ and on whether this occurrence of [10] is followed by a 0 or a 1 . Then $g \in \mathcal{G}_{+}$. Suppose that $\hat{\mu}_{+}$is a $g$-measure and let $\hat{\mu}_{-}$correspond to it under the natural bijection $M(X, T) \longrightarrow M\left(X_{-}, T_{-}\right)$. Here $X_{-}$is the space $\prod_{-\infty}^{0}\{0,1\}$. One can easily show that $\hat{\mu}_{+}\left(\left[1^{m} 00\right]\right) / \hat{\mu}_{+}\left(\left[1^{m} 0\right]\right)$ does not depends on $m$ for $m \geqslant 2$ so has a constant value $c \in(0,1)$. Hence

$$
\frac{\hat{\mu}_{-}\left(\left[1^{m} 00\right]\right)}{\hat{\mu}_{-}\left(\left[1^{m} 0\right]\right)}=\frac{\hat{\mu}_{+}\left(\left[1^{m} 00\right]\right)}{\hat{\mu}_{+}\left(\left[1^{m} 0\right]\right)}=c .
$$

Suppose that $\hat{\mu}_{-}$is a $g_{-}-$measure for some $g_{-} \in \mathcal{G}_{-}$. Then $g_{-}\left(1^{\infty} 00\right)=\hat{\mu}_{-}\left[1^{m} 00\right] /$ $\hat{\mu}_{-}\left[1^{m} 0\right]$ for all $m \geqslant 2$, so $g_{-}\left(1^{\infty} 00\right)=c$. However, one can use the properties of $\left\{d_{n}\right\}$ to show that, for each fixed $m \geqslant 1, \hat{\mu}_{+}\left(\left[0^{n} 1^{m} 0\right]\right) / \hat{\mu}_{+}\left(\left[0^{n} 1^{m} 00\right]\right) \rightarrow 0$ as $n \rightarrow \infty$. Hence $\hat{\mu}_{-}\left(\left[0^{n} 1^{m} 00\right]\right) / \hat{\mu}_{-}\left(\left[0^{n} 1^{m} 0\right]\right) \rightarrow 1$ as $n \rightarrow \infty$, so $g_{-}\left(0^{\infty} 1^{m} 00\right)=1$ for all $m \geqslant 1$. Therefore $g_{-}$cannot be continuous, because $\lim _{m \rightarrow \infty} g_{-}\left(0^{\infty} 1^{m} 00\right)=1 \neq c=$ $g_{m}\left(1^{\infty} 00\right)$.

We now show that if $g_{+} \in \mathcal{G}_{+}$and $\log g_{+} \in W(X, T)$ and $\hat{\mu}_{+}$is the unique $g_{+}$-measure, then $\hat{\mu}_{-}$is the unique $g_{-}$-measure for some $g_{-} \in \mathcal{G}_{-}$with $\log g_{-} \in$ $W\left(X_{-}, T_{-}\right)$.

TheOrem 3.6. Let $S: \hat{X} \longrightarrow \hat{X}$ be a two-sided topologically mixing subshift of finite type and let $T: X \longrightarrow X, T_{-}: X_{-} \longrightarrow X_{-}$be the corresponding one-sided topologically mixing subshifts of finite type. Let $\hat{\mu}_{+} \longrightarrow \hat{\mu}_{-}$be the natural bijection from $M(X, T)$ to $M\left(X_{-}, T_{-}\right)$described above. Let $g_{+} \in \mathcal{G}_{+}$and let $\hat{\mu}_{+}$be a $g_{+_{-}^{-}}$ measure. If $\log g_{+} \in W(X, T)$, then $\hat{\mu}_{-}$is a $g_{-}$-measure for some $g_{-} \in \mathcal{G}_{-}$and $\log g_{-} \in W\left(X_{-}, T_{-}\right)$. We have $\left|\log \left(\frac{\hat{\mu}_{-}\left(\left[x_{-n}, \ldots, x_{0}\right]\right)}{\hat{\mu}_{-}\left(\left[x_{-n}, \ldots, x_{-1}\right]\right)}\right)-\log g_{-}(x)\right| \leqslant 2 \liminf _{j \rightarrow \infty} v_{n+j}\left(T_{j} \log g_{+}\right) \quad \forall n \geqslant 1, x \in X_{-}$.

The functions $\log g_{+} \circ \pi$ and $\log g_{-} \circ \pi_{-}$are cohomologous in $C(\hat{X})$. 
Proof. For $n \geqslant 1$, define $b_{n}: X_{-} \longrightarrow(0,1)$ by

$$
b_{n}(x)=\frac{\hat{\mu}_{-}\left(\left[x_{-n}, \ldots, x_{0}\right]\right)}{\hat{\mu}_{-}\left(\left[x_{-n}, \ldots, x_{-1}\right]\right)}=\frac{\hat{\mu}_{+}\left(\left[x_{-n}, \ldots, x_{0}\right]\right)}{\hat{\mu}_{+}\left(\left[x_{-n}, \ldots, x_{-1}\right]\right)} .
$$

We show that $\left(\log b_{n}\right)$ is a Cauchy sequence in $\left(C, X_{-}\right)$. Since

$$
\frac{b_{n}(x)}{b_{n+j}(x)}=\frac{\hat{\mu}_{+}\left(\left[x_{-n}, \ldots, x_{0}\right]\right)}{\hat{\mu}_{+}\left(\left[x_{-n-j}, \ldots, x_{0}\right]\right)} \frac{\hat{\mu}_{+}\left(\left[x_{-n-j}, \ldots, x_{-1}\right]\right)}{\hat{\mu}_{+}\left(\left[x_{-n}, \ldots, x_{-1}\right]\right)},
$$

we can use Theorem 3.1 to get

$$
e^{-v_{n+j+1}\left(T_{j} \log g_{+}\right)-v_{n+j}\left(T_{j} \log g_{+}\right)} \leqslant \frac{b_{n}(x)}{b_{n+j}(x)} \leqslant e^{v_{n+j+1}\left(T_{j} \log g_{+}\right)+v_{n+j}\left(T_{j} \log g_{+}\right)} .
$$

Therefore $\left|\log b_{n}(x)-\log b_{n+j}(x)\right| \leqslant 2 v_{n+j}\left(T_{j} \log g_{+}\right)$, and since $\log g_{+} \in W(X, T)$, we have $\left(\log b_{n}\right)$ is a Cauchy sequence in $C\left(X_{-}\right)$. Hence $\log b_{n}(x) \rightrightarrows \psi(x)$ for some $\psi \in C\left(X_{-}\right)$. Since $\sum_{z \in T_{-}^{-1}(x)} b_{n}(z)=1$ for all $n \geqslant 1$, we have $\sum_{z \in T_{-}^{-1} x} e^{\psi(z)}=1$. Let $g_{-}=e^{\psi}$. Then $g_{-}$is a $g$-function for $T_{-}: X_{-} \longrightarrow X_{-}$and $\hat{\mu}_{-}$is a $g_{-}$-measure by Corollories 3.2 and 3.3 .

We get

$$
\left|\log b_{n}(x)-\log g_{-}(x)\right| \leqslant 2 \liminf _{j \rightarrow \infty} v_{n+j}\left(T_{j} \log g_{+}\right) .
$$

To see that $\log g_{-} \in W\left(X_{-}, T_{-}\right)$, we use Theorem 3.4. Since $\log g_{+} \in W(X, T)$, we have that

$$
(p, x) \longrightarrow \log \left(\frac{\hat{\mu}_{+}\left(\left[x_{0}, \ldots, x_{p+n-1}\right]\right)}{\hat{\mu}_{+}\left(\left[x_{p}, \ldots, x_{p+n-1}\right]\right)}\right)
$$

is a Cauchy sequence in $\mathrm{BC}(\mathbb{N} \times X)$. This is the equivalent to

$$
(p, z) \longrightarrow \log \left(\frac{\hat{\mu}_{-}\left(\left[z_{-(n+p-1)}, \ldots, z_{0}\right]\right)}{\hat{\mu}_{-}\left(\left[z_{-(n+p-1)}, \ldots, z_{-p}\right]\right)}\right)
$$

being a Cauchy sequence in $\mathrm{BC}\left(\mathbb{N} \times X_{-}\right)$, and hence $\log g_{-} \in W\left(X_{-}, T_{-}\right)$.

We use [15, Theorem 1.4] to see that $\log g_{+} \circ \pi$ is cohomologous to $\log g_{-} \circ \pi_{-}$ in $C(\hat{X})$. Since $\log g_{+} \in W(X, T), \log g_{+} \circ \pi$ is cohomologous in $C(\hat{X})$ to $\varphi_{-} \circ \pi_{-}$ for some $\varphi_{-} \in C\left(X_{-}\right)$. By of $\left[\mathbf{1 5}\right.$, Lemma 13], $\varphi_{-} \in W\left(X_{-}, T_{-}\right)$. Hence $\varphi_{-}$is cohomologous in $C\left(X_{-}\right)$to $\log g_{1}$ for some $g$-function $g_{1}: X_{-} \longrightarrow(0,1)$ [12]. Since $\mu_{-}$is a $g$-measure for $g_{1}$ and $g_{-}$, we have $g_{1}=g_{-}$. Hence $\log g_{+} \circ \pi$ is cohomologous to $\log g_{-} \circ \pi_{-}$in $C(\hat{X})$.

We do not know if the corresponding result holds when $W(X, T)$ is replaced by $\operatorname{Bow}(X, T)$, but we do have the following.

THEOREm 3.7. Let $S: \hat{X} \longrightarrow \hat{X}$ be a two-sided topologically mixing subshift of finite type, and let $T: X \longrightarrow X, T: X_{-} \longrightarrow X_{-}$be the corresponding one-sided topologically mixing subshifts of finite type. Let $\hat{\mu}_{+} \longrightarrow \hat{\mu}_{-}$be the natural bijection from $M(X, T)$ to $M\left(X_{-}, T_{-}\right)$described above. Let $\hat{\mu}_{+}$be a $g_{+}$-measure and $\hat{\mu}_{-}$be a $g_{-}$-measure for some $g_{+} \in \mathcal{G}_{+}$and some $g_{-} \in \mathcal{G}_{-}$. Then $\log g_{+} \in \operatorname{Bow}(X, T)$ if and only if $\log g_{-} \in \operatorname{Bow}\left(X_{-}, T_{-}\right)$.

Proof. From Theorem 3.5, we know that $\log g_{+} \in \operatorname{Bow}(X, T)$ if and only if $\hat{\mu}_{+}$ has approximate product structure, and $\log g_{-} \in \operatorname{Bow}\left(X_{-}, T_{-}\right)$if and only if $\hat{\mu}_{-}$has approximate product structure. 


\section{References}

1. T. Bousch, 'La condition de Walters', Ann. Sci. École Norm. Sup. 34 (2001) 287-311.

2. R. Bowen, 'Some systems with unique equilibrium states', Math. Systems Theory 8 (1974) 193-202.

3. R. C. Bradley, 'On the $\psi$-mixing condition for stationary random sequences', Trans. Amer. Math. Soc. 276 (1983) 55-66.

4. M. Bramson and S. Kalikow, 'Non-uniqueness of $g$-functions', Israel. J. Math. 84 (1993) 153-160.

5. N. A. Friedman, and D. Ornstein, 'On the isomorphism of weak Bernoulli transformations', Adv. Math. 5 (1970) 365-394.

6. S. KALIKOW, 'Random Markov processes and uniform martingales', Israel J. Math. 71 (1990) 33-54.

7. M. Keane, 'Strongly mixing g-measures', Invent. Math. 16 (1972) 309-324.

8. F. Ledrappier, 'Principe variationel et systèmes symbolique', Comm. Math. Phys. 33 (1973) 119-128.

9. D. Ornstein, 'On the root problem in ergodic theory', Proceedings of the Sixth Berkeley Symposium (University of California Press, 1972) 345-356.

10. A. QuAs, 'Rigidity of continuous coboundaries', Bull. London Math. Soc. 29 (1997) 595-600.

11. P. Walters, 'Ruelle's operator theorem and $g$-measures', Trans. Amer. Math. Soc. 214 (1975) 375-387.

12. P. Walters, 'Invariant measures and equilibrium states for some mappings which expand distances', Trans. Amer. Math. Soc. 236 (1978) 121-153.

13. P. Walters, An introduction to ergodic theory, Graduate Texts in Mathematics 79 (Springer, Berlin, 1982).

14. P. Walters, 'Convergence of the Ruelle operator for a function satisfying Bowen's condition', Trans. Amer. Math. Soc. 353 (2001) 327-347.

15. P. WAlters, 'A necessary and sufficient condition for a two-sided continuous function to be cohomologous to a one-sided continuous function', Dynam. Systems 18 (2003) 131-138, 271-278.

Peter Walters

Mathematics Institute

University of Warwick

Coventry CV4 7AL

United Kingdom

pw@maths.warwick.ac.uk 